\author{
Studie mit Senioren
}

\title{
Machen helle Nächte depressiv?
}

\section{Wer bei Licht im Schlafzimmer während der Nacht schläft, erhöht dadurch mög- licherweise sein Depressionsrisiko.}

_ In früheren Studien hatte sich gezeigt, dass Schlafen bei Licht mit einer Beleuchtungsstärke von fünf Lux signi- fikant mit der Entwicklung von Symptomen einer Depression assoziiert ist. Japanische Epidemiologen haben den Zusammenhang nun bei Teilnehmern der Heijo-Kyo-Kohorte überprüft.

An dieser Studie zur Gesundheit von Älteren über 60 nahmen ursprünglich mehr als 1.100 Einwohner teil. Bei ihnen wurde die LuxStärke im Schlafzimmer während der Schlafenszeit gemessen. 863 konnten an dem Follow-up von median 24 Monaten teilnehmen. Bei 710 Teilnehmern lag die Helligkeit im Schlafzimmer während der Nacht durchschnittlich bei maximal 5 Lux, bei 153 darüber.
Die Teilnehmer waren im Mittel 71,5 Jahre alt und hatten zu Studienbeginn keine Symptome einer Depression, und zwar beurteilt anhand einer Kurzfassung der Geriatric Depression Scale (GDS-15): Alle hatten zu Beginn einen Score von $<6$.

Bei Teilnehmern mit einer Helligkeit $\geq 5$ Lux während der nächtlichen Schlafenszeit hatte sich im Studienverlauf die Wahrscheinlichkeit, depressive Symptome zu entwickeln, im Vergleich zu jenen mit einem dunkleren Schlafzimmer fast verdoppelt (Hazard Ratio [HR]: 1,89; 95\%-Konfidenzintervall zwischen 1,13 und 3,14). Die Autoren vermuten, dass das Licht während der Schlafenszeit in der Nacht zu Schlafstörungen und verringerter Melatoninausschüttung führt und den zirkadianen Rhythmus stört.

$$
\text { - ple }
$$

- Obayashi Ket al. Am J Epidemiol 2017; online 31. Juli. DOl: https://doi.org/10.1093/aje/kwx290

\section{Keine Erleichterung}

\section{Prednisolon bei unteren Atemwegsinfekten nutzlos}

\section{Erwachsene ohne Asthma, die an einer akuten Infektion der unteren Atem- wege erkrankt sind, profitieren nicht von der Einnahme oraler Kortikoide, wie britische Mediziner herausgefun- den haben.}

_ Akute Infekte des unteren Atmungstrakts gehören zu den häufigsten Krankheitsbildern von Hausarztpatienten. Die Praxis, in solchen Fällen Antibiotika zu verordnen, wird mangels Nutzen und mit Blick auf möglicherweise sich entwickelnde Resistenzen viel kritisiert.
Weil die Symptome unterer Atemwegsinfekte jenen eines exazerbierten Asthmas ähneln können, sind immer wieder Versuche unternommen worden, den Patienten ihre Situation durch den Einsatz von Kortikosteroiden zu erleichtern. Die Idee dahinter ist, die entzündlich veränderte Schleimhaut zu erneuern und durch ein intaktes Epithel die Hustenrezeptoren zu schützen.

Ein Forscherteam von der Universität Bristol hat diese Hypothese nun in einer Studie überprüft. 398 ansonsten gesunde und asthmafreie Probanden mit aku- ten unteren Atemwegsinfekten, die keiner Antibiotika bedurften, bekamen über fünf Tage hinweg einmal täglich $40 \mathrm{mg}$ Prednisolon oder Placebo oral.

Ein Nutzen der Kortikoideinnahme war nicht festzustellen. Egal ob Verum oder Placebo - der Husten dauerte im Mittel fünf Tage. Die Symptomschwere auf einer Skala von 0 bis 6 lag unter Prednisolon im Mittel bei 1,99 und unter Placebo bei 2,16; diese Differenz wurde als klinisch irrelevant angesehen. 